\title{
Erratum to: Experimental and model study of the formation of chitosan-tripolyphosphate-siRNA nanoparticles
}

\author{
Tine Daa Schrøder • Yi Long • Lars Folke Olsen
}

Published online: 2 September 2014

(C) Springer-Verlag Berlin Heidelberg 2014

\section{Erratum to: Colloid Polym Sci}

\section{DOI 10.1007/s00396-014-3331-8}

The original version of the article, unfortunately, contained an error.

Table 2 was incorrectly captured. The correct table is provided below.

Table 2 Hydrodynamic diameter and polydispersity of chitosan-TPP particles formed after rapid mixing of TPP and chitosan in the presence and absence of $100 \mathrm{mM} \mathrm{NaCl}$

\begin{tabular}{|c|c|c|c|c|c|c|}
\hline \multirow[t]{4}{*}{ Treatment $^{\mathrm{a}}$} & & \multicolumn{5}{|c|}{ TPP:Glucosamine molar ratio } \\
\hline & & 0.01 & 0.06 & 0.08 & 0.10 & 0.13 \\
\hline & & \multicolumn{5}{|c|}{$[\mathrm{TPP}]_{\text {final }}(\mathrm{mM})$} \\
\hline & & 0.04 & 0.21 & 0.29 & 0.37 & 0.46 \\
\hline \multirow[t]{2}{*}{$100 \mathrm{mM} \mathrm{NaCl}$} & $\mathrm{D}_{\mathrm{H}}(\mathrm{nm})^{\mathrm{b}}$ & $11 \pm 5^{\mathrm{d}}$ & $571 \pm 37$ & $434 \pm 69$ & $451 \pm 8$ & $514 \pm 54$ \\
\hline & $\mathrm{PD}^{\mathrm{c}}$ & $0.650 \pm 0.276$ & $0.092 \pm 0.009$ & $0.141 \pm 0.008$ & $0.175 \pm 0.005$ & $0.227 \pm 0.013$ \\
\hline \multirow[t]{2}{*}{$\mathrm{No} \mathrm{NaCl}$} & $\mathrm{D}_{\mathrm{H}}(\mathrm{nm})^{\mathrm{b}}$ & $7 \pm 1^{\mathrm{e}}$ & $42 \pm 4$ & $51 \pm 8$ & $53 \pm 9$ & $59 \pm 8$ \\
\hline & $\mathrm{PD}^{\mathrm{c}}$ & n. d. & $0.220 \pm 0.044$ & $0.164 \pm 0.050$ & $0.163 \pm 0.014$ & $0.138 \pm 0.018$ \\
\hline
\end{tabular}

n. $d$. Not determined

${ }^{a}$ The particles were made by a single injection of TPP into a chitosan solution $(1 \mathrm{mg} / \mathrm{ml})$ as described in Measurements of kinetics of particle formation. The hydrodynamic diameter $\left(\mathrm{D}_{\mathrm{H}}\right)$ and polydispersity $(\mathrm{PD})$ were measured on a Brookhaven instrument

${ }^{\mathrm{b}}$ The listed $\mathrm{D}_{\mathrm{H}}$ are given by volume distribution and with standard deviation

${ }^{\mathrm{c}} \mathrm{PD}$ is intensity-weighted and given with standard deviation

${ }^{\mathrm{d}}{ }_{n=2}$

${ }^{\mathrm{e}}$ Only in two out of three samples could particles be detected by dynamic light scattering

The online version of the original article can be found at http://dx.doi.org/ 10.1007/s00396-014-3331-8.

T. D. Schrøder $(\bowtie) \cdot$ Y. Long $\cdot$ L. F. Olsen

Celcom, Department of Biochemistry and Molecular Biology,

University of Southern Denmark, Campusvej 55, 5230 Odense M,

Denmark

e-mail: tids@bmb.sdu.dk 